\title{
Polyatomic Molecules Formed with a Rydberg Atom in an Ultracold Environment
}

\author{
Ivan C. H. Liu* and Jan M. Rost \\ Max-Planck Institute for the Physics of Complex Systems, Nöthnitzer Str. 38, 01187 Dresden, Germany
}

\begin{abstract}
We investigate properties of ultralong-range polyatomic molecules formed with a Rb Rydberg atom and several ground-state atoms whose distance from the Rydberg atom is of the order of $n^{2} a_{0}$, where $n$ is the principle quantum number of the Rydberg electron. In particular, we put emphasis on the splitting of the energy levels, and elucidate the nature of the splitting via the construction of symmetry-adapted orbitals.
\end{abstract}

\section{INTRODUCTION}

The recent advancement of ultracold physics has made possible the study of many interesting phenomena, ranging from the formation of molecules in a Bose-Einstein Condensate (BEC) [1, 2, 3] to correlation effects in ultracold neutral plasmas [4, 5], where in the former case the atoms are cooled to temperature in the nano-Kelvin range. Combined with narrow bandwidth lasers and high resolution spectroscopy [6], new phenomena can be studied involving high-lying Rydberg states which have a narrow spacing in energy of the order of $10 \mathrm{GHz}$. One such example is the theoretical prediction of the formation of ultralong-range dimers by a Rydberg atom and a nearby ground-state atom [7], the so-called "trilobite" molecules. The potential well supporting the vibrational bound states is extremely weak compared to typical ground-state molecules. The depth of well is $\sim 15 \mathrm{GHz}$ for $n=30$, where $n$ is the principle quantum number of the Rydberg atom, and scales as $n^{-3}$. The long-range nature of such system, bound at the equilibrium distance of the order of $10^{3}$ a.u., is rather unusual as well as the oscillatory feature at the bottom of the potential well. This class of ultralong-range dimers should be distinguished from another kind, due to the Rydberg-Rydberg interaction [8], whose molecular resonance was observed in the experiments [9, 10].

In this paper we address the question if more than one ground-state atom can form, together with the Rydberg atom, a polyatomic molecule. We find, that shape and symmetry of such polyatomic molecules follow a systematics which is well understandable on the one hand side in terms of trilobite building blocks, that is, linear combinations of wavefunctions which describe the Rydberg atom and a single ground-state atom. From a more global perspective, these polyatomic molecules can be classified according to irreducible representations reflecting their symmetry properties, as it is well known in quantum chemistry.

So far, trilobites have not been identified experimentally. As the experimentally achieved density of ultracold atomic ensembles increases [11], so does the likelyhood

\footnotetext{
*Electronic address: ivanliu@pks.mpg.de
}

${ }^{\dagger}$ Electronic address: rost@pks.mpg.de of detecting such molecular species. However, there are still open questions concerning the realizability of such a molecule under current experimental conditions. For example, the Rydberg electron is very likely to interact with multiple ground-state perturbers, and it is not so clear at first sight what the role of the Rydberg states is. Such scenarios will also concern the proposed "dipole blockade" scheme for quantum information processing [12]. The characterization of polyatomic molecules involving one Rydberg and several ground-state atoms helps to understand the possible role of such ground-state perturber better.

Technically speaking, we extend the calculations done by Greene, Dickinson and Sadeghpour 7] to include multiple ground-state atoms using the Fermi pseudopotential treatment. More sophisticated methods exist 13, 14], but the results do not differ much, whilst the qualitative features are certainly captured which suffice for the purpose of the present article. Using the Fermi pseudo-potential also allows one to calculate easily a large system.

We investigate, in particular, the effect of placing the perturbers in a structured environment on the splitting of the adiabatic energy levels of the molecular system. We will use group theory to obtain the total wavefunction of the system in the framework of first-order perturbation theory via the construction of the symmetry-adapted orbitals. For the sake of clarity we restrict the investigation to atoms all lying in a plane (whereas the Rydberg electron of course lives in the physical 3D space). Atomic units are used unless stated otherwise.

\section{THE HAMILTONIAN}

First, we consider a ground-state atom with label $i$ located at distance $R_{i}$ from the Rydberg atom. The ground-state atom influences the electron by its polarization field, which has the form $-\alpha / 2 r^{4}$, where $\alpha$ is the atomic polarizability. For Rb atoms, the experimentally determined value is $\alpha=319.2$ [15]. To a good approximation, the potential - extremely short-ranged with respect to the extension of a Rydberg electronic wavefunction - can be mimiced by the Fermi pseudo-potential, namely [16],

$$
\hat{V}_{i}=2 \pi L\left[k_{i}\right] \delta\left(\vec{r}-\vec{R}_{i}\right)
$$


where $L\left[k_{i}\right] \equiv-\left(\tan \delta_{s}\right) / k_{i}$ is the s-wave energydependent scattering length of the Rydberg electron colliding with a neutral atom; $k_{i}^{2} / 2=-1 / 2 n^{2}+1 / R_{i}$ its kinetic energy, and $\vec{r}$ its distance from the mother ion. The s-wave phase shift $\delta_{s}$ can be calculated using the modified effective range theory by O'Malley et al [17] and the zero-energy scattering length for triplet s-wave calculated by Bahrim et al [18]. The singlet scattering length is much smaller, and hence we do not expect it to influence the phenomena discussed here.

In the case of $\mathrm{Rb}$ atoms, the quantum defect is negligible for high $l$-states $(l \geqslant 3)$, which are quasi-denergate. They are therefore well represented by hydrogenic wavefunctions. The low- $l$ states, on the other hand, split away from the $n$-manifold and do not interact with the high$l$ states provided that the energy-dependent scattering length is sufficiently small. The high- $l$ states are also more interesting, because the Hilbert space is larger in this case, which produces a more flexible system, i.e. the different eigenstates are allowed to interfere with each other. This results in a highly-polarizable complex. In this paper, we consider only the high- $l$ class.

The total Hamiltonian of the Rydberg electron interacting with its mother ion and $N$ ground-state atoms can be written as

$$
\hat{H}=\hat{H}_{0}+\hat{V}_{N}
$$

where the ionic Hamiltonian is

$$
\hat{H}_{0}=\frac{\hat{p}^{2}}{2}-\frac{1}{r} .
$$

The potential $V_{N}$ is the $N$-fold sum over the interaction Eq. (11) with all ground-state atoms, i.e.,

$$
\hat{V}_{N}=2 \pi \sum_{i=1}^{N} L\left[k_{i}\right] \delta\left(\vec{r}-\vec{R}_{i}\right),
$$

where $i$ labels the $i$-th ground-state atom, and $N$ is the total number of ground-state atoms.

The effect of $p$-wave electron scattering was neglected in our calculation, however, in the dimer case it was studied by Hamilton et al [19] and Khuskivadze et al [14].

\section{DETERMINATION OF ADIABATIC ENERGY CURVES}

Under the adiabatic approximation, the energy levels calculated from the Hamiltonian $\hat{H}$ (including the perturbation) give, automatically, the electronic structure of the molecular system involving $N+1$ atoms. We determine a cut through the Born Oppenheimer (BO) potential surface for systems with $N=2,3$, and 4 ground state atoms which are uniformly placed on a circle with radius $R$ centered at the Rydberg core. These three cases correspond to linear, triangular, and square geometries, respectively. The cut we choose corresponds to the breathing mode, i.e. $R$ is varied. We calculate the $\mathrm{BO}$ curves

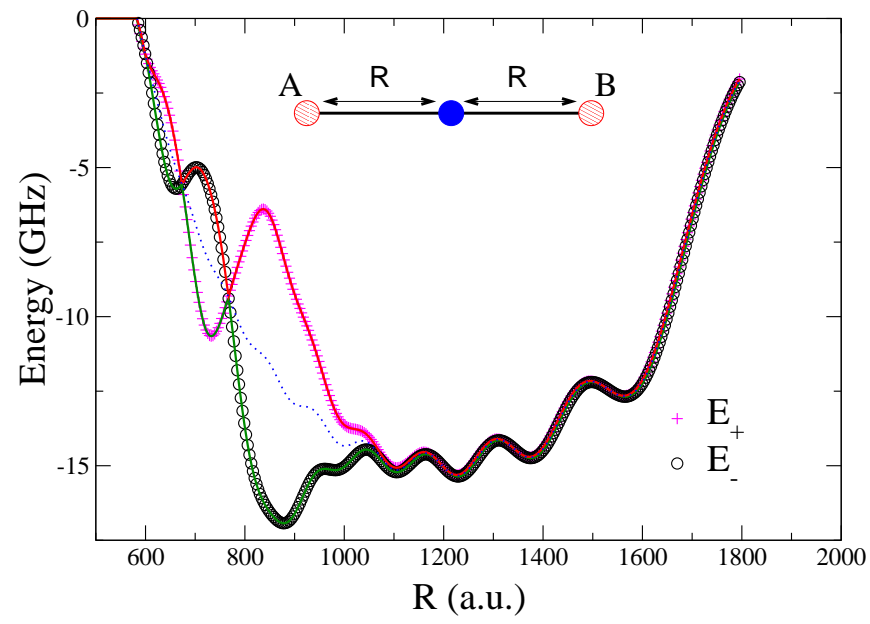

FIG. 1: Adiabatic energy curves for the linear diatomic (dotted line) and triatomic molecule (solid lines) as a function of distance $R$ between Rydberg and ground-state atoms, see also the sketch for the triatomic molecule with the Rydberg atom in the middle. The solid lines are the results from diagonalization, and the points $(+)$ and (o) are the enegry expectation values calculated from the symmetry-adapted orbitals Eq. (6) and (7), respectively. The $E=0$ lies at the energy of the $n=30$ manifold.

using two methods: (i) the direct diagonalisation of $\hat{H}$; and (ii) the projection operator method 20] to construct symmetry-adapted orbitals and determine the BO curves from standard perturbation theory. Both methods are accurate, but the latter gives a deeper insight into the quantum mechanical properties such as the energy degeneracy.

The Fermi pseudo-potential is usually valid for $n \gtrsim 25$ 30. For smaller principle quantum numbers, the scattering of $e^{-}+\mathrm{Rb}$ and the polarization of the neutral perturber by the Rydberg atomic core are not independent of each other [21]. Here, we present calculations for $n=30$, which also allows us to compare our results directly with that previously obtained for the $\mathrm{Rb}_{2}$ dimer [7, 14].

The eigenvalues from the unperturbed Hamiltonian, $\hat{H}_{0}$, yields simply the hydrogenic energy $E_{0}=-1 / 2 n^{2}$, so for convenience we set this to be zero throughout this article.

\section{COLLINEAR TRIATOMIC MOLECULE $(\mathrm{N}=2)$}

Consider two ground-state atoms (A and B) placed on either side of a Rydberg atom with distances $R$ forming a collinear triatomic molecule. This configuration corresponds to $N=2$, and the numerical result of the $\mathrm{BO}$ curves are plotted in Fig. 1.

In order to understand the splitting of the energy levels, we use the perturbed state $\left|\psi_{n}(\vec{r})\right\rangle$ when only one of the two ground-state atoms is present as the building 
block for constructing the total electronic wavefunction. The perturbed state can be can be explicitly written as [22]

$$
\left|\psi_{n}(\vec{r})\right\rangle=\sum_{q} \phi_{n q}^{*}(\vec{R})\left|\phi_{n q}(\vec{r})\right\rangle
$$

where the index $q$ runs over all the degenerate states which includes all $l$ 's and $m$ 's with $l \geq 3$. We call this wavefunction the "trilobite" wavefunction since it produces the probability density like that drawn in Ref. [7]. The two wavefunctions, which clearly satisfy the parity of the collinear geometry, are

$$
\left|\psi_{+}(\vec{r})\right\rangle=\left|\psi_{n}^{A}(\vec{r})\right\rangle+\left|\psi_{n}^{B}(\vec{r})\right\rangle,
$$

and

$$
\left|\psi_{-}(\vec{r})\right\rangle=\left|\psi_{n}^{A}(\vec{r})\right\rangle-\left|\psi_{n}^{B}(\vec{r})\right\rangle,
$$

where the superscript $A$ and $B$ are the labels of the ground-state atoms; $\left|\psi_{n}^{A}(\vec{r})\right\rangle$ and $\left|\psi_{n}^{B}(\vec{r})\right\rangle$ are the trilobite wavefunctions when only atom $\mathrm{A}$ or $\mathrm{B}$ is present. By choosing the projection axis $\hat{z}$ so that it aligns with the internuclear axis, the only degenerate states that contribute are those with non-zero value along $\hat{z}$. They are in this case the states with $m=0$.

Using the above ansatz to calculate the expectation value $\left\langle\hat{V}_{N}\right\rangle$ yields immediately two energies $E_{+}$and $E_{-}$, which are distinguished by their parities:

$$
E_{ \pm}(R)=L[k] \sum_{l=3}^{n-1}(2 l+1)\left|u_{n l}(R)\right|^{2}\left\{\begin{array}{l}
l=\text { even for } E_{+} \\
l=\text { odd for } E_{-}
\end{array}\right.
$$

where $L[k]=L\left[k_{A}\right]=L\left[k_{B}\right], R=\left|\vec{R}_{A}\right|=\left|\vec{R}_{B}\right|$ and $u_{n l}$ is the radial part of the hydrogenic wavefunction $\phi_{n l}(\vec{R})=$ $u_{n l}(R) Y_{l m}(\theta, \varphi)$.

Hence, we see that, with the inclusion of the second perturber, two curves split away from the $n$-manifold, with one corresponding to gerade and the other to ungerade symmetry. They both converge at large distance to the curve when only one ground-state atom is present. However, they split from each other approximately within $R / r_{n} \leq 1$ (with $r_{n}=n^{2}=900$ ), which can be seen from the sum of the probability densities of even- $l$ and odd- $l$ states. They differ when the overlap $\left\langle\psi_{n}^{B} \mid \psi_{n}^{A}\right\rangle$ is not exponentially small, see Fig. 2. This feature is general for all principle quantum numbers. The additional splitting also suggests that the system can be more stable with the inclusion of more than one neutral perturber, a situation we will investigate in more detail in Section V.

In Fig. 3, we show the contour plot of the probability density of the diatomic and triatomic systems at the interatomic distance $R_{m}=879$, which corresponds to the deepest potential energy. The special minimum configuration can be most clearly identified by means of the

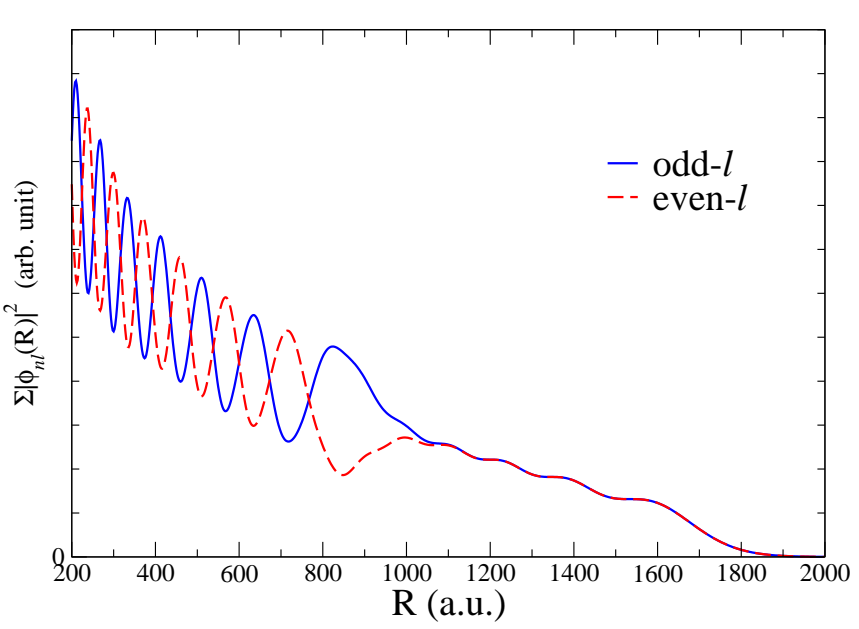

FIG. 2: Sum of probability densities $\sum_{l}\left|\phi_{n l}(\vec{R})\right|^{2}$ for states with even and odd angular momentum components $l$ as a function of the radial distance.

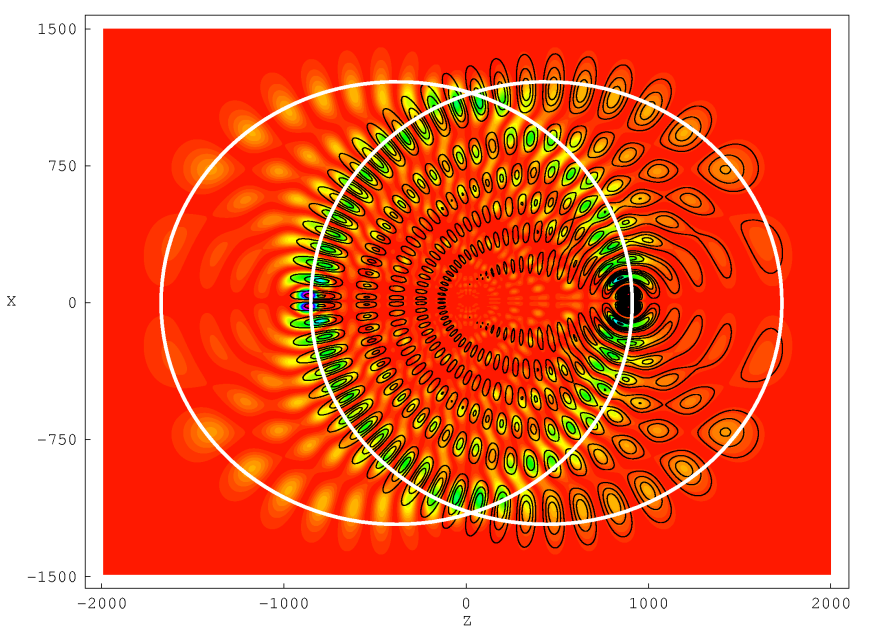

FIG. 3: Cut of the electronic probability density along the internuclear axis at the deepest point of the potential well, $R=879$. The black contour lines show the probibility density of the trilobite (diatomic) wavefunction, and the background coloured plot is for the $N=2$ (collinear triatomic) configuration. The ground-state atoms are located at $(x, z)=(0, \pm 879)$ and the Rydberg atom at $(0,0)$. The two white solid lines show the classical Kepler ellipses.

classical Kepler orbits along which the two trilobite states of the molecule are scarred (see also Ref. [23]). Each Kepler ellipse has one ground-state atom in one of its foci and touches the other ground-state atom. 


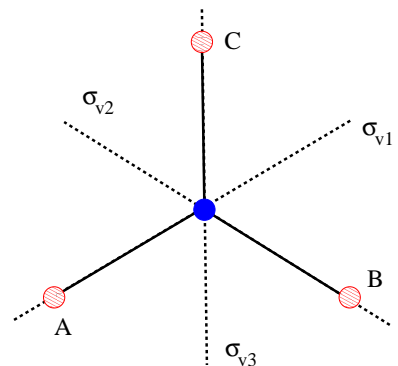

(a)

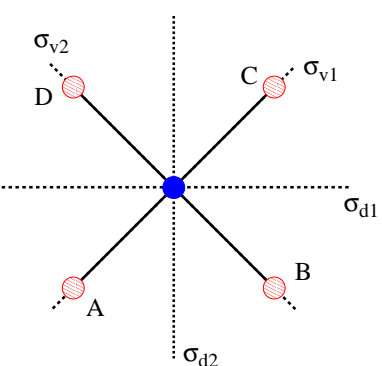

(b)
FIG. 4: Illustration of the geometry of the (a) $C_{3, v}$ and (b) $C_{4, v}$ configurations. The dashed lines are the planes of reflection. The shaded and the solid circles are the ground-state and the Rydberg atoms respectively.

\section{PLANAR POLYATOMIC MOLECULES OF TRIANGULAR AND QUADRATIC SHAPE $(\mathrm{N}=3,4)$}

For $N \geq 3$, we choose $\hat{z}$ to be perpendicular to the plane containing the atoms. Figure 4 illustrates the spacial geometry of the complexes. The degenerate states that contribute, i.e., the index $q$ in Eq. (5), are the states with $l+m$ being an even integer, exclusive of $l=0,1,2$ states. The results are shown in Fig. 5 .

For the $N=3$ case, three energy eigenvalues split away from the $n$-manifold, and as in the case of $N=2$, beyond $r_{n}$ they converge to the BO curve for the dimer case. Note, however, that two of the energy levels are degenerate at any given distance within $r_{n}$, indicating that there are additional symmetries preserved under the perturbation of the ground-state atoms. To elucidate these symmetries, we again use the trilobite state as ba-

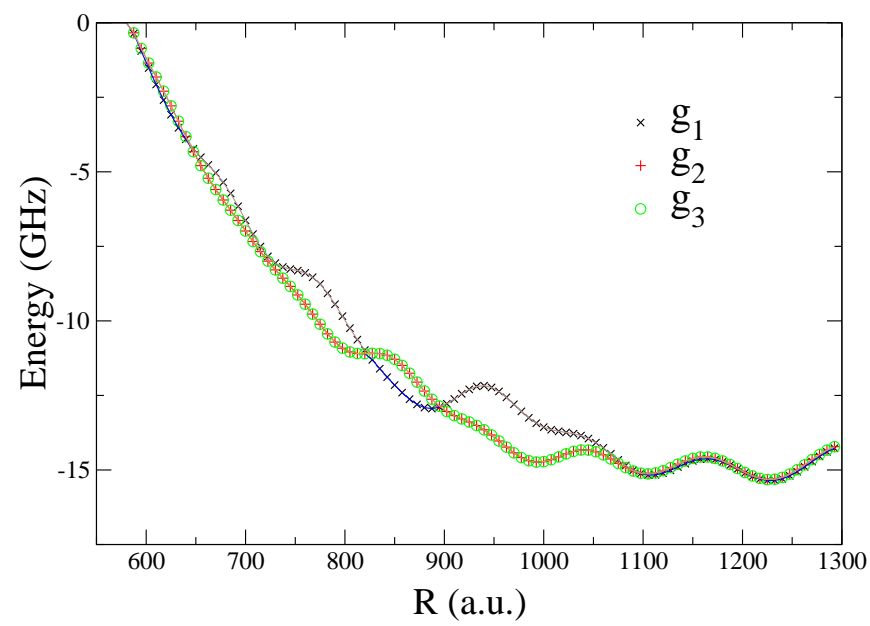

FIG. 5: The three adiabatic energy curves $g_{i}$ for a triangular configuration as a function of Rydberg-ground state atomic distance $R$ (see Fig. $4 \mathrm{a}$ ). The coding of the data is analogous to Fig. 1 .
TABLE I: Character tables of the irreducible representations of (a) $C_{3, v}$ and (b) $C_{4, v}$ [25]. The labeling follows the conventional rules.

(a)

(b)

\begin{tabular}{|c|c|c|c|c|c|c|c|c|c|}
\hline & $\mathrm{E}$ & $C_{3}$ & $\sigma_{v}$ & & $\mathrm{E}$ & & $2 C_{4}$ & $2 \sigma_{v}$ & $2 \sigma_{d}$ \\
\hline$\Gamma_{1}$ & 1 & 1 & 1 & $\overline{\Gamma_{1}}$ & 1 & 1 & 1 & 1 & 1 \\
\hline$\Gamma_{2}$ & 1 & 1 & -1 & $\Gamma_{2}$ & 1 & 1 & 1 & -1 & -1 \\
\hline \multirow[t]{3}{*}{$\Gamma_{3}$} & 2 & -1 & 0 & $\Gamma_{3}$ & 1 & 1 & -1 & 1 & -1 \\
\hline & & & & $\Gamma_{4}$ & 1 & 1 & -1 & -1 & 1 \\
\hline & & & & $\Gamma_{5}$ & 2 & -2 & 0 & 0 & 0 \\
\hline
\end{tabular}

sis functions to construct the relevant symmetry-adapted orbitals. But unlike in the previous collinear configuration, where taking account of the parity as the relevant symmetry is intuitive, we have to use a systematic approach for $N=3$ or larger. A method which has been used extensively to find the symmetry-adapted orbitals is the projection operator method, which is expressed mathematically as [20]

$$
g=\frac{l_{j}}{h} \sum_{\hat{R}} \chi_{j}^{*}(\hat{R}) \hat{O}_{R} f,
$$

where $\hat{O}_{R}$ is the operator for a particular symmetry operation $\hat{R}$, e.g., rotation or reflection, etc.. The $l_{j}$ and $\chi_{j}$ are, respectively, the dimension and the character of the $j$-th irreducible representation (irrep) of the symmetry group, to which the system belongs, while $h$ is the order of the group. The sum extends over all symmetry operations in the group. This equation allows us to find the symmetry-adapted functions $\left\{g_{i}\right\}$ from any original basis set $\left\{f_{i}\right\}$. The general proof of this proceedure can be found, for example, in section 6.6 of Ref. [24].

The name of this procedure originates from the fact that the pre-factor in front of $f$ in the above equation can be viewed as a projection operator that projects the basis set $\left\{f_{i}\right\}$ into a basis set $\left\{g_{i}\right\}$ that diagonalizes the Hamiltonian matrix. In other words, $g$ and $f$ in Eq. (9) are vectors, and the operator

$$
\hat{P}_{\Gamma} \equiv \frac{l_{j}}{h} \sum_{\hat{R}} \chi_{j}^{*}(\hat{R}) \hat{O}_{R}
$$

can be represented by a unitary matrix.

The configurations of $N=3$ and 4 correspond to the symmetry groups $C_{3, v}$ and $C_{4, v}$, and their character tables of the irreducible representations are shown in Table I.

We find that in the $N=3$ case, the representation of the symmetry operations using the trilobite state as the basis set contains only two of the total three irreducible representations, $\Gamma_{1}$ and $\Gamma_{3}$ (see Table $\mathrm{I}(\mathrm{a})$ and Appendix A 1 for details), which are one- and two-dimensional respectively. The symmetry-adapted orbitals constructed will 


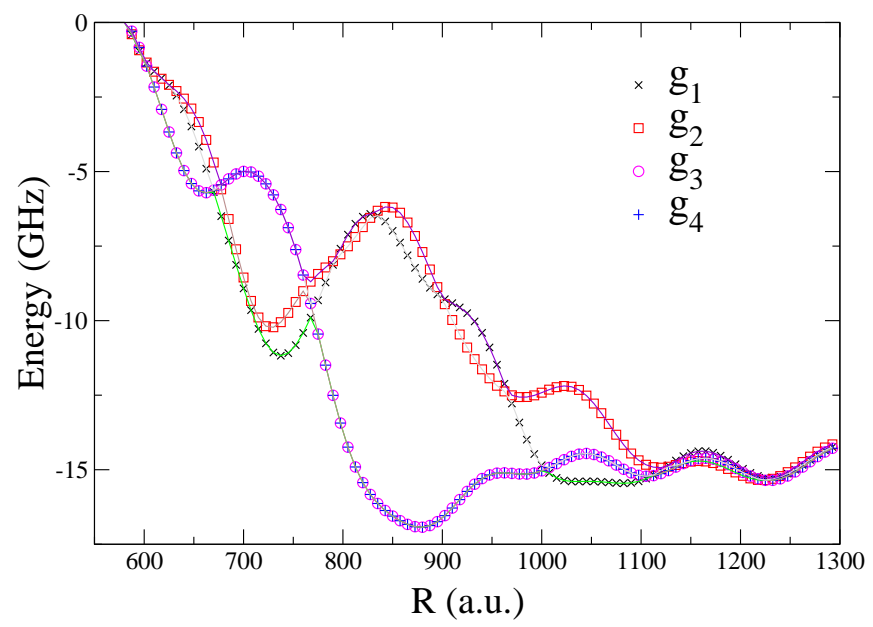

FIG. 6: Same as Fig. 5] but for a square geometry with a ground-state atom at each corner (see Fig. 40) with four energy curves $g_{i}$, see text.

then, according to the fundamental theory of quantum mechanics, consist of a non-degenerate and two degenerate states. They are, repectively, $g_{1}, g_{2}$ and $g_{3}$ shown explicitly in Eq. (A5), A6a) and (A7) in Appendix A1.

The energy expectation values,

$$
\left\langle\hat{V}_{N}(R)\right\rangle_{g_{i}} \equiv\left\langle g_{i}\left|\hat{V}_{N}(R)\right| g_{i}\right\rangle,
$$

calculated using the $g_{i}$-functions $i=1,2,3$ are plotted in Fig. 5. As expected, the two curves belonging to $\Gamma_{3}$ overlap with each other at all distances $R$. Note that applying $\hat{P}_{\Gamma_{2}}$ onto any of the basis functions produces zero, which is a general feature when the irrep is not contained in the overall representation.

The same analysis for the $C_{4, v}$ symmetry reveals that the overall representation contains $\Gamma_{1}, \Gamma_{3}$, and $\Gamma_{5}$ (see Table. $\mathrm{I}(\mathrm{b})]$, with the first being one-dimensional, and the second and the third two-dimensional (see Appendix A2 for detail). Hence, there are two sets of doublydegenerate $\mathrm{BO}$ curves and a non-degenerate one. Applying Eq. (9), we obtain the symmetry-adapted orbitals $g_{i}, i=1,2,3,4$ as shown in Eq. (A11)-(A14) in Appendix A2 The adiabatic energy levels from the analytical and numerical results are plotted in Fig. 6. Again, the graph shows a perfect agreement between the two results.

\section{CONCLUSION}

We have used the Fermi pseudo-potential to model the effect neutral $\mathrm{Rb}$ ground-state atoms have on a Rydberg electron. Cuts through the resulting potential surface, adiabatic in the distance of the ground-state perturbers from the ionic core of the Rydberg electron, have been calculated for different arrangements of the groundstate atoms which form planar polyatomic molecules. We found that more ground-state atoms lead to more deeply bound molecules compared to the original diatom as studied by Greene and coworkers [7]. A systematic understanding of the structure and symmetry of such molecules can be gained by taking the trilobite (diatomic) wavefunction as a basic unit and constructing symmetryadapted orbitals as demonstrated in Section IV and V. For two ground-state atoms the procedure is relative simple and intuitive, while three or more ground-state atoms require a systematic approach, such as the projection operator method.

In the case where $N$ is larger than the number of degenerate states $q_{\max }$, our method of constructing the perturbed wavefunction should still work, and will yield only $q_{\text {max }}$ linearly independent states.

The effect of $p$-wave electron scattering plays an important role especially in hydrogen-like atoms. Our calculations can be extended into the case of higher partial wave scattering by using the appropriate pseudo-potentials formulated by Omont [22]. Previous studies [14, 19] for dimers show that the potential curve of the $p$-wave scattering crosses the potential well at $R \approx 1200$, and could potentially destabilize the trilobite while also providing additional potential wells. It will be also interesting to see how the spatial arrangement of the atoms affect the energy of the system in this process, since the potential is now dependent on the gradient of the electronic wavefunction in the 3D space.

The present work is a first exploration of the possibility to form polyatomic molecules from a single Rydberg atom and a number of ground-state atoms. We have only determined a cut (at equal distances of the ground-state atoms to the Rydberg atom) through the multidimensional potential surface which resembles the potential for the breathing mode of the molecule. Future analysis and realistic assessment of the quantitative features of such species must include the vibrational motion of the atoms.

\section{APPENDIX A: DERIVATION OF THE SYMMETRY-ADAPTED ORBITALS}

\section{Planar Polyatomic Molecule with $\mathrm{N}=3$}

The molecule formed in this configuration has the symmetry of the point group $C_{3, v}$. Using trilobite states as the basis set to contruct the corresponding representation, one obtains the following matrices,

$$
\begin{aligned}
\hat{O}_{E}=\left(\begin{array}{lll}
1 & 0 & 0 \\
0 & 1 & 0 \\
0 & 0 & 1
\end{array}\right) & \hat{O}_{C_{3}^{1}}=\left(\begin{array}{lll}
0 & 1 & 0 \\
0 & 0 & 1 \\
1 & 0 & 0
\end{array}\right) \\
\hat{O}_{C_{3}^{2}}=\left(\begin{array}{lll}
0 & 0 & 1 \\
1 & 0 & 0 \\
0 & 1 & 0
\end{array}\right) & \hat{O}_{\sigma_{v 1}}=\left(\begin{array}{lll}
1 & 0 & 0 \\
0 & 0 & 1 \\
0 & 1 & 0
\end{array}\right) \\
\hat{O}_{\sigma_{v 2}}=\left(\begin{array}{lll}
0 & 0 & 1 \\
0 & 1 & 0 \\
1 & 0 & 0
\end{array}\right) & \hat{O}_{\sigma_{v 3}}=\left(\begin{array}{lll}
0 & 1 & 0 \\
1 & 0 & 0 \\
0 & 0 & 1
\end{array}\right)
\end{aligned}
$$


In the above notations, $E$ is the identity; $C_{k}^{p}$ denotes the rotation about $\hat{z}$-axis by angle $2 \pi p / k$; and the $\sigma$ 's are the relfections through the planes perpendicular to the plane of the atoms, as indicated in Fig. 4(a).

It is clear that when one of the above operators, say $\hat{O}_{C_{3}^{1}}$, is applied on the original vector, the result is a $120^{\circ}$ rotation about $\hat{z}$ in the counter-clockwise direction, assuming that $\hat{z}$ is pointing perpendicularly into the paper, i.e.

$$
\left(\begin{array}{lll}
0 & 1 & 0 \\
0 & 0 & 1 \\
1 & 0 & 0
\end{array}\right)\left(\begin{array}{c}
\psi_{n}^{A} \\
\psi_{n}^{B} \\
\psi_{n}^{C}
\end{array}\right) \longrightarrow\left(\begin{array}{c}
\psi_{n}^{B} \\
\psi_{n}^{C} \\
\psi_{n}^{A}
\end{array}\right) .
$$

From Eq. (A1), the character of the representation in such basis set can be determined by taking the trace of each matrix, and they are summarized in the table below:

$$
\begin{array}{c|ccc} 
& \mathrm{E} & 2 C_{3} & 3 \sigma_{v} \\
\hline \Gamma_{\text {total }} & 3 & 0 & 1
\end{array}
$$

Here, we have used $\Gamma_{\text {total }}$ to denote the representation formed by the trilobite states. By inspecting the character table of the irreps of $C_{3, v}$ (Table $\bar{I}(\mathrm{a})$, , one immediately sees that the current representation is a direct sum of $\Gamma_{1}$ and $\Gamma_{3}$, namely,

$$
\Gamma_{\text {total }}=\Gamma_{1} \oplus \Gamma_{3} .
$$

Now, we determine the projection operators in each irrep by using Eq. (10). The order of the group is $h=6$, and the dimensions for $\Gamma_{1}$ and $\Gamma_{3}$ are $l_{1}=1$ and $l_{3}=2$, respectively. Equation (10) then yields

$$
\begin{aligned}
& \hat{P}_{\Gamma_{1}}=\frac{1}{6}\left[\hat{O}_{E}+\hat{O}_{C_{3}^{1}}+\hat{O}_{C_{3}^{2}}+\hat{O}_{\sigma_{v 1}}+\hat{O}_{\sigma_{v 2}}+\hat{O}_{\sigma_{v 3}}\right], \\
& \hat{P}_{\Gamma_{2}}=\frac{1}{6}\left[\hat{O}_{E}+\hat{O}_{C_{3}^{1}}+\hat{O}_{C_{3}^{2}}-\hat{O}_{\sigma_{v 1}}-\hat{O}_{\sigma_{v 2}}-\hat{O}_{\sigma_{v 3}}\right], \\
& \hat{P}_{\Gamma_{3}}=\frac{1}{6}\left[2 \hat{O}_{E}+\hat{O}_{C_{3}^{1}}+\hat{O}_{C_{3}^{2}}\right] .
\end{aligned}
$$

Since $\Gamma_{\text {total }}$ contains only $\Gamma_{1}$ and $\Gamma_{3}$, we need to apply only Eq. (A4a) and (A4c) to our basis set in order to obtain the symmetry-adapted orbitals. Acting the trivial operator $\hat{P}_{\Gamma_{1}}$ on the trilobite wavefunction $\left|\psi_{n}^{A}\right\rangle$, we obtain the first symmetry-adapted orbital

$$
\hat{P}_{\Gamma_{1}} f_{A}=\hat{P}_{\Gamma_{1}}\left|\psi_{n}^{A}\right\rangle=\frac{1}{3}\left[\left|\psi_{n}^{A}\right\rangle+\left|\psi_{n}^{B}\right\rangle+\left|\psi_{n}^{C}\right\rangle\right] \equiv g_{1} .
$$

The same equations are obtained if one acts $\hat{P}_{\Gamma_{1}}$ on $\left|\psi_{n}^{B}\right\rangle$ or $\left|\psi_{n}^{C}\right\rangle$ which are obviously linearly-dependent. However, acting $\hat{P}_{\Gamma_{3}}$ on $\left|\psi_{n}^{A}\right\rangle,\left|\psi_{n}^{B}\right\rangle$ and $\left|\psi_{n}^{C}\right\rangle$ gives, repectively,

$$
\begin{aligned}
\hat{P}_{\Gamma_{3}}\left|\psi_{n}^{A}\right\rangle & =\frac{1}{6}\left[2\left|\psi_{n}^{A}\right\rangle-\left|\psi_{n}^{B}\right\rangle-\left|\psi_{n}^{C}\right\rangle\right] \equiv g_{2}, \\
\hat{P}_{\Gamma_{3}}\left|\psi_{n}^{B}\right\rangle & =\frac{1}{6}\left[2\left|\psi_{n}^{B}\right\rangle-\left|\psi_{n}^{C}\right\rangle-\left|\psi_{n}^{A}\right\rangle\right], \\
\hat{P}_{\Gamma_{3}}\left|\psi_{n}^{C}\right\rangle & =\frac{1}{6}\left[2\left|\psi_{n}^{C}\right\rangle-\left|\psi_{n}^{B}\right\rangle-\left|\psi_{n}^{A}\right\rangle\right] .
\end{aligned}
$$

Since $\Gamma_{3}$ is a three-dimensional irrep, two of the above equations can be combined, by subtracting Eq. (A6b) by (A6c), giving

$$
\frac{1}{6}\left[\left|\psi_{n}^{B}(\vec{r})\right\rangle-\left|\psi_{n}^{C}(\vec{r})\right\rangle\right] \equiv g_{3},
$$

so that finally we have three linearly-independent wave functions, which we call $g_{1}, g_{2}$ and $g_{3}$.

\section{Planar Polyatomic Molecule with $\mathrm{N}=4$}

Following the same procedure as in the case of $N=3$, one finds the matrices of the symmetry operations in the point group $C_{4, v}$ in the present basis set as,

$$
\begin{aligned}
& \hat{O}_{E}=\left(\begin{array}{cccc}
1 & 0 & 0 & 0 \\
0 & 1 & 0 & 0 \\
0 & 0 & 1 & 0 \\
0 & 0 & 0 & 1
\end{array}\right) \quad \hat{O}_{C_{2}^{1}}=\left(\begin{array}{llll}
0 & 0 & 1 & 0 \\
0 & 0 & 0 & 1 \\
1 & 0 & 0 & 0 \\
0 & 1 & 0 & 0
\end{array}\right) \\
& \hat{O}_{C_{4}^{1}}=\left(\begin{array}{cccc}
0 & 1 & 0 & 0 \\
0 & 0 & 1 & 0 \\
0 & 0 & 0 & 1 \\
1 & 0 & 0 & 0
\end{array}\right) \quad \hat{O}_{C_{4}^{3}}=\left(\begin{array}{llll}
0 & 0 & 0 & 1 \\
1 & 0 & 0 & 0 \\
0 & 1 & 0 & 0 \\
0 & 0 & 1 & 0
\end{array}\right) \\
& \hat{O}_{\sigma_{v 1}}=\left(\begin{array}{cccc}
1 & 0 & 0 & 0 \\
0 & 0 & 0 & 1 \\
0 & 0 & 1 & 0 \\
0 & 1 & 0 & 0
\end{array}\right) \quad \hat{O}_{\sigma_{v 2}}=\left(\begin{array}{llll}
0 & 0 & 1 & 0 \\
0 & 1 & 0 & 0 \\
1 & 0 & 0 & 0 \\
0 & 0 & 0 & 1
\end{array}\right) \\
& \hat{O}_{\sigma_{d 1}}=\left(\begin{array}{cccc}
0 & 1 & 0 & 0 \\
1 & 0 & 0 & 0 \\
0 & 0 & 0 & 1 \\
0 & 0 & 1 & 0
\end{array}\right) \quad \hat{O}_{\sigma_{d 2}}=\left(\begin{array}{cccc}
0 & 0 & 0 & 1 \\
0 & 0 & 1 & 0 \\
0 & 1 & 0 & 0 \\
1 & 0 & 0 & 0
\end{array}\right)
\end{aligned}
$$

where the notations are as before, and the planes of reflections are indicated in Fig. 4(b)

The character of $\Gamma_{\text {total }}$ can again be determined by taking the trace of each matrix above, and they are:

$$
\begin{array}{c|ccccc} 
& \mathrm{E} & C_{2} & 2 C_{4} & 2 \sigma_{v} & 2 \sigma_{d} \\
\hline \Gamma_{\text {total }} & 4 & 0 & 0 & 2 & 0
\end{array}
$$

Again, from the character table of the irrep (Table $\mathrm{I}(\mathrm{b})$, one finds that the representation $\Gamma_{\text {total }}$ is a direct sum of

$$
\Gamma_{\text {total }}=\Gamma_{1} \oplus \Gamma_{3} \oplus \Gamma_{5} .
$$

Hence, we know that in this representation there are two one-dimensional and one two-dimensional irreps. Their corresponding projection operators can be obtained by applying Eq. (10), where in this case, $h=8$, and $l_{1}, l_{3}$ and $l_{5}$ are 1,1 and 2 , repectively. Therefore, the projection 
operators are

$$
\begin{aligned}
\hat{P}_{\Gamma_{1}}= & \frac{1}{8}\left[\hat{O}_{E}+\hat{O}_{C_{2}^{1}}+\hat{O}_{C_{4}^{1}}+\hat{O}_{C_{4}^{2}}\right. \\
& \left.+\hat{O}_{\sigma_{v 1}}+\hat{O}_{\sigma_{v 2}}+\hat{O}_{\sigma_{d 1}}+\hat{O}_{\sigma_{d 2}}\right], \\
\hat{P}_{\Gamma_{3}}= & \frac{1}{8}\left[\hat{O}_{E}+\hat{O}_{C_{2}^{1}}-\hat{O}_{C_{4}^{1}}-\hat{O}_{C_{4}^{2}}\right. \\
& \left.+\hat{O}_{\sigma_{v 1}}+\hat{O}_{\sigma_{v 2}}-\hat{O}_{\sigma_{d 1}}-\hat{O}_{\sigma_{d 2}}\right], \\
\hat{P}_{\Gamma_{5}}= & \frac{1}{4}\left[\hat{O}_{E}-\hat{O}_{C_{2}^{1}}\right] .
\end{aligned}
$$

The symmetry-adapted orbitals can then be obtained in a similar way as in Appendix A1, which yields the following four linearly-independent equations:

$$
\begin{aligned}
& g_{1}=\frac{1}{4}\left[\left|\psi_{n}^{A}(\vec{r})\right\rangle+\left|\psi_{n}^{B}(\vec{r})\right\rangle+\left|\psi_{n}^{C}(\vec{r})\right\rangle+\left|\psi_{n}^{D}(\vec{r})\right\rangle\right], \\
& g_{2}=\frac{1}{4}\left[\left|\psi_{n}^{A}(\vec{r})\right\rangle-\left|\psi_{n}^{B}(\vec{r})\right\rangle+\left|\psi_{n}^{C}(\vec{r})\right\rangle-\left|\psi_{n}^{D}(\vec{r})\right\rangle\right] \\
& g_{3}=\frac{1}{4}\left[\left|\psi_{n}^{A}(\vec{r})\right\rangle-\left|\psi_{n}^{C}(\vec{r})\right\rangle\right] \\
& g_{4}=\frac{1}{4}\left[\left|\psi_{n}^{B}(\vec{r})\right\rangle-\left|\psi_{n}^{D}(\vec{r})\right\rangle\right]
\end{aligned}
$$

[1] R. Wynar, R. S. Freeland, C. R. D. J. Han, and D. J. Heinzen, Science 287, 1016 (2000).

[2] J. M. Gerton, D. Strekalov, I. Prodan, and R. G. Hulet, Nature 408, 692 (2000).

[3] C. McKenzie, J. H. Denschlag, H. Häffner, A. Browaeys, L. E. E. de Araujo, F. K. Fatemi, K. M. Jones, J. E. Simsarian, D. Cho, A. Simoni, et al., Phys. Rev. Lett. 88, 120403 (2002).

[4] T. C. Killian, S. Kulin, S. D. Bergeson, L. A. Orozco, C. Orzel, and S. L. Rolston, Phys. Rev. Lett. 83, 4776 (1999).

[5] T. Pohl, T. Pattard, and J. M. Rost, Phys. Rev. Lett. 92, 155003 (2004).

[6] W. Li, I. Mourachko, M. W. Noel, and T. F. Gallagher, Phys. Rev. A 67, 052502 (2003).

[7] C. H. Greene, A. S. Dickinson, and H. R. Sadeghpour, Phys. Rev. Lett. 85, 2458 (2000).

[8] C. Boisseau, I. Simbotin, and R. Côté, Phys. Rev. Lett. 88, 133004 (2002).

[9] S. M. Farooqi, D. Tong, S. Krishnan, J. Stanojevic, Y. P. Zhang, J. R. Ensher, A. S. Estrin, C. Boisseau, R. Côté, E. E. Eyler, et al., Phys. Rev. Lett. 91, 183002 (2003).

[10] L. G. Marcassa, A. L. de Oliveira, M. Weidemüller, and V. S. Bagnato, Phys. Rev. A 71, 054701 (2005).

[11] J. Sebby-Strabley, R. T. R. Newell, J. O. Day, E. Brekke, and T. G. Walker, Phys. Rev. A 71, 021401(R) (2005).

[12] M. D. Lukin, M. Fleischhauer, R. Côté, L. Duan,
D. Jaksch, J. I. Cirac, and P. Zoller, Phys. Rev. Lett. 87, 037901 (2001).

[13] M. I. Chibisov, A. A. Khuskivadze, and I. I. Fabrikant, J. Phys. B: At. Mol. Opt. Phys. 35, L193 (2002).

[14] A. A. Khuskivadze, M. I. Chibisov, and I. I. Fabrikant, Phys. Rev. A 66, 042709 (2002). erson, Phys. Rev. A 10, 1131 (1974).

[16] E. Fermi, Nuovo Cimento 11, 157 (1934).

[17] T. F. O'Malley, L. Spruch, and L. Rosenberg, J. Math. Phys. 2, 491 (1961).

[18] C. Bahrim, U. Thumm, and I. I. Fabrikant, J. Phys. B: At. Mol. Opt. Phys. 34, L195 (2001).

[19] E. L. Hamilton, C. H. Greene, and H. R. Sadeghpour, J. Phys. B: At. Mol. Opt. Phys. 35, L199 (2002).

[20] I. N. Levine, Molecular Spectroscopy (John Wiley and Sons, Inc., 1975).

[21] I. I. Fabrikant, J. Phys. B: At. Mol. Opt. Phys. 19, 1527 (1986).

[22] A. Omont, J. de Physique 38, 1343 (1977).

[23] B. R. Granger, E. L. Hamilton, and C. H. Greene, Phys. Rev. A 64, 042508 (2001).

[24] D. S. Schonland, Molecular Symmetry (Van Nostrand, Princeton, New Jersey, 1965).

[25] J. A. Salthouse and M. J. Ware, Point Group Character Tables (Cambridge University Press, 1972).
[15] R. W. Molof, H. L. Schwartz, T. M. Miller, and B. Bed- 